\title{
展望硼烯的化学合成
}

杨金龙

中国科学技术大学化学与材料科学学院, 合肥 230026

\section{An Outlook of Chemical Synthesis of Borophenes}

\author{
YANG Jinlong
}

School of Chemistry and Materials Science, University of Science and Technology of China, Hefei 230026, P. R. China.

Email: jlyang@ustc.edu.cn

二维材料因独特的尺度具备一系列宏观材料 所未有的物理和化学性质, 相关研究不仅充分展 示了材料世界的鬼米力, 而且为制造下一代电子器 件提供了重要科学与技术基础。二维材料研究的 热点之一是寻找新的具有更独特结构和物性的单 原子层材料, 以丰富整个二维材料的家族。自发现 石墨烯之后, 国际上已报道了数百种二维材料, 它 们展现的晶格结构和物性可谓多姿多彩。然而, 令 人惊讶的是, 像石墨烯这样具有纯平面原子结构 且由单一元素组成的二维材料一直未见报道。直 到2016年, 二维硼单层-嗍烯-的出现材料改变了这 一局面; 它是由美国西北大学与阿贡国家实验室 联合课题组 ${ }^{1}$ 和中国科学院物理研究所吴克辉课 题组 ${ }^{2}$ 分别在超高真空条件下, 在干净的银表面上 通过分子束外延技术沉积高纯度嗍源制备得到。

需要指出的是, 硼烯的理论研究早在上个世 纪末就已开始 3 。然而, 由于硼烯没有对应的层状 体块结构, 实验进展缓慢, 使得理论研究在接下来 的十几年里成为该领域的主角。理论研究者对嗍 烯的研究由零维团簇外推到二维结构, 由孤立体 系扩展到基底状态, 并提出了如何合成二维硼烯 的可行途径 ${ }^{4}$ 。经过理论研究的充分积累, 硼烯最 近被上述两个实验室成功制备。较为罕见的是, 实 验表征发现, 银基底上的嗍烯结构与早期理论所 预测的原子结构完全一致 ${ }^{5}$, 完美诠释了理论研究 在新材料发现中的重要指导作用。同时, 硼烯实现 过程中这种理论与实验的成功配合为制备其它合 成二维材料提供了良好借鉴。

不同于石墨烯的半金属性, 硼烯具有金属性,
是目前报道的最轻、最薄的金属体 ${ }^{6}$ 。另外, 嗍烯 在力学性质方面上是集坚、柔、韧于一体的纳米材 料7, 具有广泛的工程应用前景。然而, 喼烯的合 成依然是该领域的一大挑战。当前实验制备嗍烯 还仅限于昂贵的分子束外延方法, 所得样品尺寸 也非常有限。为促进硼烯的实际应用, 实现在基底 上化学气相沉积(CVD)生长大面积高品质的嗍烯 样品是必经之路。鉴于此, 南京航空航天大学张助 华教授等人围绕硼烯制备方面的理论与实验研究 进展进行了系统分析和总结, 展望了在特定基底 上化学气相沉积合成嗍烯的可能性。

该综述已在物理化学学报上在线发表 (doi: 10.3866/PKU.WHXB201805080) ${ }^{8}$ 。该文系统分析 了生长基底、温度和前驱物等重要因素对硼烯成 核和生长的影响, 探讨了嗍烯可能的生长机制及 所涉及的理论研究方法, 以期能够帮助实验者通 过基底选择、生长条件控制等手段实现化学气相 沉积制备嗍烯。该文对实现通过化学合成制备大 面积、高质量的硼烯样品有启发作用。

\section{References}

(1) Mannix, A. J.; Zhou, X. F.; Kiraly, B.; Wood, J. D.; Alducin, D.; Myers, B. D.; Liu, X. L.; Fisher, B. L.; Santiago, U.; Guest, J. R.; et al. Science 2015, 350, 1513. doi: 10.1126/science.aad 1080

(2) Feng, B. J.; Zhang, J.; Zhong, Q.; Li, W. B.; Li, S.; Li, H.; Cheng, P.; Meng, S.; Chen, L.; Wu, K. H. Nat. Chem. 2016, 8, 563. doi: 10.1038/NCHEM.2491

(3) Boustani, I.; Quandt, A.; Hernandez, E.; Rubio, A. J. Chem. Phys. 1999, 110, 3176. doi: 10.1063/1.477976 
(4) Zhang, Z. H.; Yang, Y.; Penev, E. S.; Yakobson, B. I. Chem. Soc. Rev. 2017, 46, 6746. doi: 10.1039/c7cs00261k

(5) Zhang, Z. H.; Yang, Y.; Gao, G. Y.; Yakobson, B. I. Angew. Chem. Int. Ed. 2015, 54, 13022. doi: 10.1002/anie.201505425

(6) Tang, H.; Ismail-Beigi, S. Phys. Rev. Lett. 2007, 99, 115501. doi: 10.1103/PhysRevLett.99.115501
(7) Zhang, Z. H.; Yang, Y.; Penev, E. S.; Yakobson, B. I. Adv. Funct. Mater. 2017, 27, 1605059. doi: 10.1002/adfm.201605059

(8) Wang, Q.; Xue, M.; Zhang, Z. Acta Phys. -Chim. Sin. 2019, 35 (6), 565. [王琴, 薛敏珢, 张助华. 物理化学学报, 2019, 35 (6), 565.] doi: 10.3866/PKU.WHXB201805080 